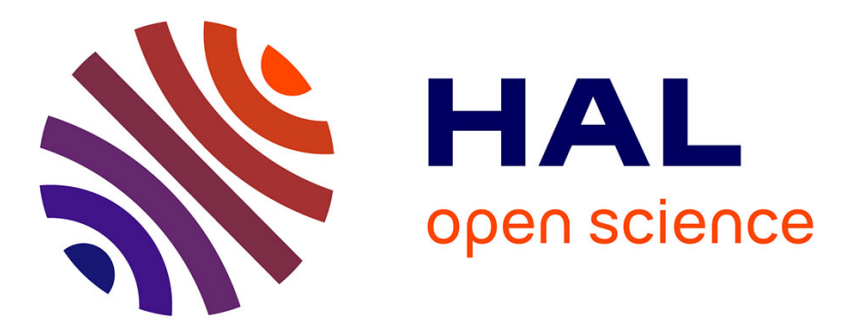

\title{
High photoluminescence of shortwave infrared-emitting anisotropic surface charged gold nanoclusters
}

Benjamin Musnier, David Karl Wegner, Clothilde Comby-Zerbino, Vanessa

Trouillet, Muriel Jourdan, Ines Häusler, Rodolphe Antoine, Jean-Luc Coll,

Ute Resch-Genger, Xavier Le Guével

\section{To cite this version:}

Benjamin Musnier, David Karl Wegner, Clothilde Comby-Zerbino, Vanessa Trouillet, Muriel Jourdan, et al.. High photoluminescence of shortwave infrared-emitting anisotropic surface charged gold nanoclusters. Nanoscale, 2019, 11 (25), pp.12092-12096. 10.1039/c9nr04120f . hal-02353153

\section{HAL Id: hal-02353153 \\ https://hal.science/hal-02353153}

Submitted on 7 Nov 2019

HAL is a multi-disciplinary open access archive for the deposit and dissemination of scientific research documents, whether they are published or not. The documents may come from teaching and research institutions in France or abroad, or from public or private research centers.
L'archive ouverte pluridisciplinaire HAL, est destinée au dépôt et à la diffusion de documents scientifiques de niveau recherche, publiés ou non, émanant des établissements d'enseignement et de recherche français ou étrangers, des laboratoires publics ou privés. 


\section{High Photoluminescence of Shortwave Infrared-emitting Anisotropic Surface Charged Gold Nanoclusters}

Received 00th January 20xx, Accepted 00th January 20xx

DOI: $10.1039 / \times 0 x \times 00000 x$

\section{Benjamin Musnier ${ }^{a \ddagger}$, K. David Wegner ${ }^{\mathrm{b} \ddagger}$, Clothilde Comby-Zerbino ${ }^{c}$, Vanessa Trouillet ${ }^{d}$, Muriel} Jourdan ${ }^{\mathrm{e}}$, Ines Häusler ${ }^{f}$, Rodolphe Antoine ${ }^{c}$, Jean-Luc Coll ${ }^{{ }^{*}}$, Ute Resch-Genger ${ }^{{ }^{*}}$, Xavier Le Guével $^{\mathrm{a}^{*}}$
Incorporating anisotropic surface charges on atomically precise gold nanoclusters (Au NCs) led to a strong absorption in the nearinfrared and could generate the formation of self-assembled $\mathrm{Au}$ NCs exhibiting an intense absorption band at $\sim 1000 \mathrm{~nm}$. This surface modification showed a striking enhancement of the photoluminescence in the Shortwave Infrared (SWIR) region with a quantum yield as high as $6.1 \%$ in water.

Compared to the NIR I window (600-900 $\mathrm{nm})$, the shortwave infrared window (SWIR, 900-1700 nm) is a promising spectral window for bioimaging due to the reduced autofluorescence and scattering of tissue ${ }^{1}$. Recent imaging studies demonstrated for instance a significant improvement in the spatial and temporal resolution of mouse vasculature in the SWIR ${ }^{2-5}$ However, few emitters are suitable for this spectral window. Single-wall carbon nanotubes and organic dyes exhibit rather weak photoluminescence (PL) above $1000 \mathrm{~nm}^{6,7}$, and InAs- and Ag2S-based quantum dots, which show relatively high quantum yields $(P L Q Y s)^{5}, 8,9$, raise concerns regarding their toxicity hampering in vivo applications. In 2017, it was shown that $\mathrm{Au}$ NCs stabilized by lipoic-acid-based sulfobetaine ligands reveal a broad emission with a PL maximum at approximately $900 \mathrm{~nm}$ and a surprisingly high PL QY of 3.8\%, enabling detection above $1100 \mathrm{~nm}^{10}$. In the search for promising SWIR contrast agents

a. B. Musnier, JL. Coll*, X. Le Guevel*, Institute for Advanced Biosciences, University of Grenoble Alpes/INSERM1209/CNRS-UMR5309-Grenoble-France xavier.le-guevel@univ-grenoble-alpes; jean-luc.coll@univ-grenoble-alpes.fr

b. K.D. Wegner, U. Resch-Genger*, BAM Federal Institute for Materials Research and Testing, Richard-Willstaetter-Str.11,12489 Berlin, Germany ute.resch@bam.de

c. C. Comby-Zerbino, R. Antoine, Institut lumière matière, UMR5306, Université Claude Bernard Lyon1-CNRS, Univ. Lyon 69622 Villeurbanne cedex, France

d.V. Trouillet, Institute for Applied Materials (IAM) and Karlsruhe Nano Micro Facility (KNMF), Karlsruhe Institute of Technology (KIT), Eggenstein-Leopoldshafen, Germany

e.M. Jourdan, Université Grenoble Alpes, Département de Chimie Moléculaire (DCM)-CNRS UMR5250, Grenoble, France

f. Ines Häusler, Technische Universität Berlin, Institut für Optik und Atomare Physik, Straße des 17. Juni 135, 10623 Berlin, Germany

¥ authors equally contributed to this work

Electronic Supplementary Information (ESI) available: [details of any supplementary information available should be included here]. See DOI: 10.1039/x0xx00000x with high PL QYs, we introduced a short dithiol pegylated molecule onto atomically precise Au NCs and investigated how the pairing affects the optical properties of the resulting clusters.

a

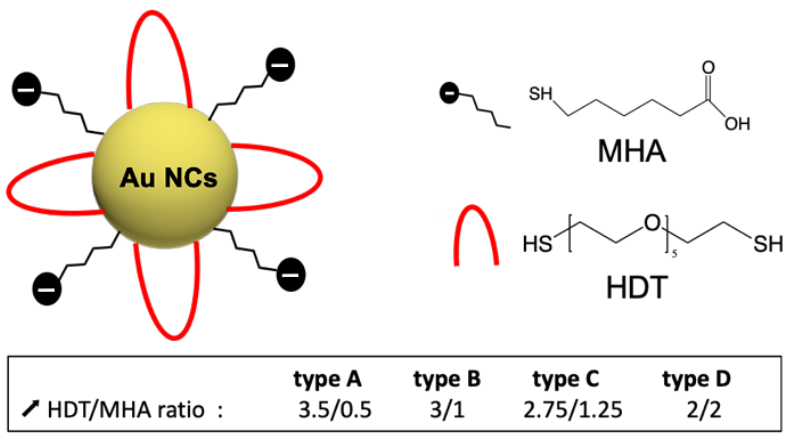

b
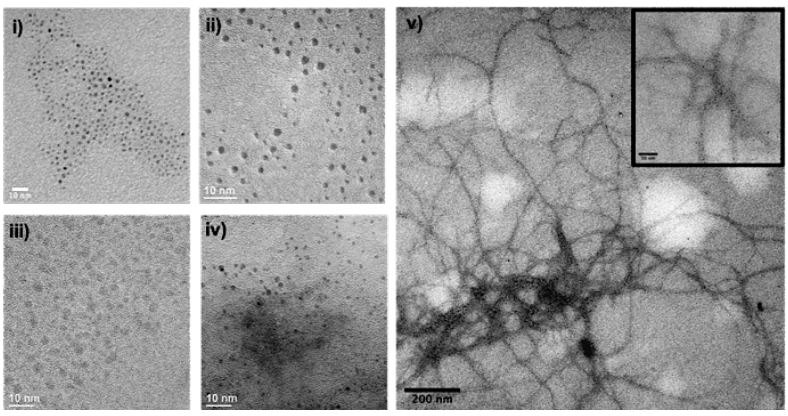

Figure 1. (a) Scheme of Au NC synthesis. (b) High-resolution TEM images of (i) AuMHA, (ii) AuMHA/HDT type A, (iii) AuMHA/HDT type B, (iv) AuMHA/HDT type C, and (v) AuMHA/HDT type D.

From single NCs to self-assembly. A series of $A u$ NCs were synthesized by a bottom-up approach using $\mathrm{HAuCl}_{4} \cdot 3 \mathrm{H}_{2} \mathrm{O}$ and different molar ratios of monodentate mercaptohexanoic acid (MHA) and the bidentate ligand hexa(ethylene glycol) dithiol (HDT) in alkaline solution (see Experimental section in SI). We prepared five types of $\mathrm{Au}$ NCs denoted as AuMHA and AuMHA/HDT type A, B, C, and D for which the amount of HDT was increased during synthesis (Figure 1a). High-resolution transmission electron microscopy (HRTEM) (Figure 1b; Figs. S1- 
5) confirmed the ultrasmall size of the Au NCs, with gold cores below $3 \mathrm{~nm}$, that possessed a semicrystalline structure. As shown in Figure $\mathbf{1 b}(\mathbf{v})$, the Au NCs obtained with the highest HDT concentration (AuMHA/HDT type D) exhibited selfassembly of the individual clusters into a dendritic network of micrometer dimensions. The metal cores, as determined by TEM, were similar in size $\sim 1.6 \mathrm{~nm}$ for all Au NC series with good monodispersity (see Table S1). The hydrodynamic diameter of the $\mathrm{Au}$ NC types with the atomically precise characteristic (AuMHA and AuMHA/HDT types A and B) was obtained by diffusion-ordered spectroscopy (DOSY) (Fig. S6). The slight growth in size from $1.54 \pm 0.04 \mathrm{~nm}$ from AuMHA to $2.46 \pm 0.20 \mathrm{~nm}$ for AuMHA/HDT type B was attributed to the increased hydrophobicity and steric hindrance of HDT (Table S1).

The composition of the Au NCs was characterized by X-ray photoelectron spectroscopy (XPS) and by electrospray mass spectrometry (ESI-MS). The concentration of (C-O) originating from HDT, as obtained from XPS measurements, was used to determine the HDT/MHA ratio at the Au NC's surface (Fig. S7). The atomic ratio ( $\mathrm{C}-\mathrm{O}) /(\mathrm{C}-\mathrm{C}, \mathrm{C}-\mathrm{H})$ was directly correlated to the $\mathrm{HDT} / \mathrm{MHA}$ ratio, and it increased consistently with the amount of HDT used in the cluster synthesis, with AuMHA/HDT type $C$ having the highest concentration. The drop in HDT/MHA for the Au NCs with the highest HDT concentration (AuMHA/HDT type D) indicated a surface saturation leading to the self-assembly of $\mathrm{Au}$ NCs via sharing of one or more dithiol ligands. The $\mathrm{Au}_{n} \mathrm{MHA}_{m} \mathrm{HDT}_{p}$ stoichiometry for Au NCs presenting atomic precision was then evaluated utilizing the average mass estimated from the deconvoluted ESI mass spectra (Fig. S8) and the different atomic molar ratios derived from XPS measurements (see SI methods and Fig. S7). These analyses yielded a stoichiometry of $\mathbf{A u}_{25} \mathbf{M H A}_{18}$ for AuMHA. We proposed possible stoichiometries of $\mathbf{A u}_{23} \mathbf{M H A}_{18} \mathbf{H D T}_{6}$ and $\mathrm{Au}_{23} \mathrm{MHA}_{17} \mathrm{HDT}_{9}$ for the main populations of type $A$ and type $B$ systems, respectively. A second minor population was observed for types $A$ and $B$ and was ascribed to $\mathbf{A u}_{25} \mathbf{M H A}_{19} \mathbf{H D T}_{10}$. Similar measurements with type $C$ revealed a species with a higher molecular weight, indicating the onset of HDT saturation per $\mathrm{Au}$ NC.

Optical properties of Au NCs with anisotropic surface charge. HDT incorporation in AuMHA NCs drastically modified their photophysical properties. Absorption measurements with AuMHA (see Figure 2 a) revealed a main absorption band at 670 $\mathrm{nm}$ and two shoulders at 440 and $770 \mathrm{~nm}$. This spectral pattern is typical for thiolated $\mathrm{Au}_{25} \mathrm{NCs}^{11}$. Adding a small amount of HDT (AuMHA/HDT type A) led to the disappearance of the $440 \mathrm{~nm}$ absorption band and a shift of the $670 \mathrm{~nm}$ band to $690 \mathrm{~nm}$, while the $770 \mathrm{~nm}$ peak became more pronounced. Additionally, new bands at $610 \mathrm{~nm}$ and $983 \mathrm{~nm}$ appeared. Further HDT additions enhanced the NIR bands at $776 \mathrm{~nm}$ and $988 \mathrm{~nm}$ by factors of 2.3 and 6.6 and introduced a $\sim 5 \mathrm{~nm}$ red shift (see Figure $\mathbf{2 b}$ ). Similar changes in absorption were also observed in an earlier study ${ }^{12}$ using the co-ligand 2,2' (ethylenedioxy)diethanethiol (EDT) and were ascribed to the presence of two differently charged ligands (MHA - negative, EDT - neutral) leading to a surface charge anisotropy, although the exact mechanism was unclear. The key feature of EDT and HDT is their bidentate nature, with both terminal thiol groups being able to bind to the Au NC surface. This can cause a structural distortion in thiolated Au NCs, which strongly affects their photophysical properties. These absorption bands seem similar to $\mathrm{Au}_{25} \mathrm{NC}$ species rather than $\mathrm{Au}_{23} \mathrm{NC}^{12,13}$. However, considering the presence of both $\mathrm{Au}_{23} \mathrm{NC}$ and $\mathrm{Au}_{25} \mathrm{NC}$ in type $\mathrm{A}$ and type $B$ and with the increase of $A u$ complexes in single $A u$ NCs confirmed by XPS, there is certainly a surface contribution to the absorbance profile. We cannot exclude that the presence of bidentate ligands may induce changes in the kernel motif which may also affect the absorption spectra. The peak at approximately $780 \mathrm{~nm}$ was previously attributed to a chargevariation-induced structural distortion caused by HDT, which explained the augmentation of the peak with increasing HDT concentrations (see Figure 2a). The peak at approximately 980 $\mathrm{nm}$ can result from micro-distortions of the Au NCs surface induced by surface charge anisotropy.
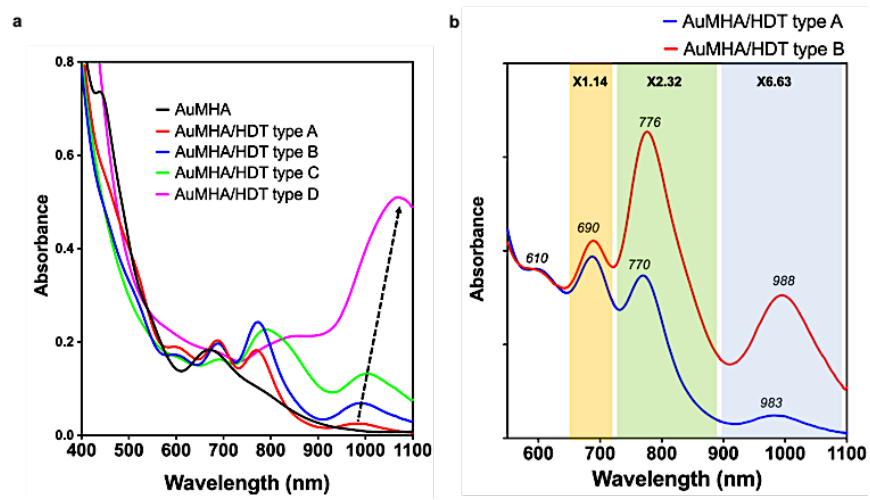

Figure 2. (a) Absorption spectra of AuMHA and AuMHA/HDT types A-D in water between 400 and $1100 \mathrm{~nm}$. (b) Absorption spectra of AuMHA/HDT type $A$ and type B normalized at $610 \mathrm{~nm}$. Each color band corresponds to an optical window where the surface area below each curve is compared.

In contrast to the previous study, we investigated the influence of high HDT concentrations corresponding to the type C system, which showed a broadened and bathochromic-shifted absorption band at $800 \mathrm{~nm}$. Such shift seems to be in agreement with the presence of multimeric Au NC species such as dimers and trimers ${ }^{13}, 14$. Surprisingly, for the highest HDT concentration used in this study (type D), a large red shift to $1080 \mathrm{~nm}$ was observed for the longest-wavelength absorption band. This translation was accompanied by a strong increase in absorption intensity by a factor of 35 over that of AuMHA (see Figure 2a). We excluded a plasmonic contribution arising from the size of our metal core being below $3 \mathrm{~nm}^{15}$. To confirm that the increased absorption intensities at $780 \mathrm{~nm}$ and $980 \mathrm{~nm}$ originated from a surface charge anisotropy, we measured the absorption spectra of these Au NCs at different $\mathrm{pH}$ values from 4.5 to 9.0 (Fig. S9), thereby tuning the ligand charge by (de)protonating the MHA's carboxylic acid group. For type A, this modification affected the ratio of the $670 \mathrm{~nm}$ and $780 \mathrm{~nm}$ absorption bands, with the latter being enhanced at lower $\mathrm{pH}$ values, indicating a change in the structural distortion. For higher HDT concentrations (type B and type C), however, no protonation-induced change in the absorption was observed. This could be attributed to a reduced charge effect with the pegylated chain of HDT protecting Au NC surface to the environment. The disappearance of the $980 \mathrm{~nm}$ band at $\mathrm{pH} 4.5$ for both types of Au NCs confirmed our hypothesis that the structural and optical effects were related to a surface charge anisotropy. The case was different for type $D$, where saturation of the Au NCs' surface with HDT resulted in self-assembly 
structure confirmed by HRTEM (Fig. S5) and ESI-MS, where ESIMS spectra of AuMHA/HDT type D were drastically different from those recorded for AuMHA/HDT type A and type B (see Fig. S8). Here, we observed only a red shift in the $1080 \mathrm{~nm}$ absorption for type D. Similarly, to their counterpart plasmonic NPs, this shift might be attributed to a long-range dipole coupling in the $\mathrm{Au} N \mathrm{NC}$ assembly ${ }^{16}$. Apparently, Au NC selfassembly led to micro-distortions that could not be reversed by protonation. Therefore, these effects cannot be solely attributed to surface charge anisotropy. Multimeric Au NC species have been also studied theoretically by Whetten's team ${ }^{17}$ and by Maran's lab ${ }^{18}$. They showed that Au NCs could switch between metallic and semi-conducting properties when they start to self-assemble at long distances in one dimension. Similar optical properties were obtained for Au NCs with smaller dithiol pegylated ligands (Fig. S10), with the confirmation that NIR absorption for self-assembled Au NC structure could be obtained with cross-linkers of different length. However, HDT provided the sharpest and most intense NIR absorption bands and further investigations both from theoretical and experimental sides are necessary to fully understand their photonic properties.

Ultrasmall gold nanomaterials exhibit optical features of great interest for imaging applications thanks to their PL up to or even exceeding $1000 \mathrm{~nm}$ and to their long PL lifetime in the microsecond range ${ }^{19,} 20$. The PL of these metal systems is commonly ascribed to an energy transfer within the metal core and from a metal surface partially influenced by surface ligands, as demonstrated experimentally and theoretically ${ }^{21-24}$. Studies of the PL properties of our series of Au NCs at different excitation wavelengths $(400 \mathrm{~nm}, 780 \mathrm{~nm}$, and $980 \mathrm{~nm}$ ) revealed a weak PL of AuMHA at $900 \mathrm{~nm}$ and a broad shoulder that originated from another PL band located at $1030 \mathrm{~nm}$, as shown in Figure 3a.
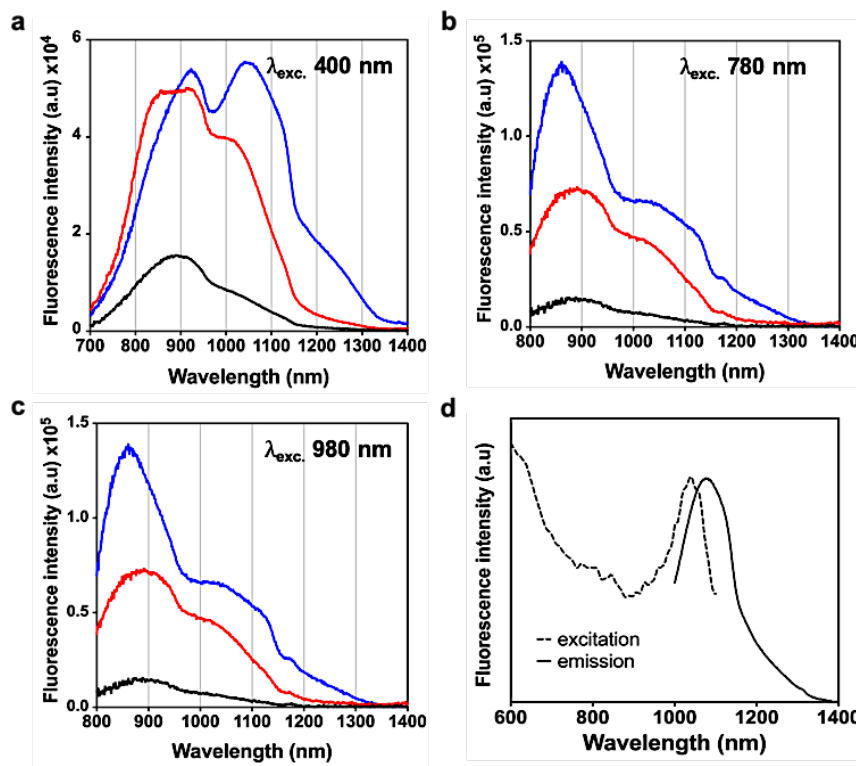

Figure 3. (a-c) Photoluminescence spectra of AuMHA (black), AuMHA/HDT type A (red), and AuMHA/HDT type B (blue) between 700 and $1400 \mathrm{~nm}$ at different excitation wavelengths $(400 \mathrm{~nm}, 780 \mathrm{~nm}$, and $980 \mathrm{~nm}$ ). (d) Normalized excitation/emission spectra of AuMHA/HDT type B. Measurements were performed in water.
The PL profile of AuMHA/HDT type A was similar but enhanced by a factor of 6.8 compared to that of AuMHA. The emission band of AuMHA/HDT type B was strongly modified resulting in distinct bands at $932 \mathrm{~nm}$ and $1075 \mathrm{~nm}$ with shoulders at 1125 $\mathrm{nm}$ and $1225 \mathrm{~nm}$, respectively. An increase in the HDT content of the Au NCs clearly affected their PL spectra. Deconvolution of the PL spectra demonstrated that the largest contributions arose from PL bands at $1125 \mathrm{~nm}$ and $1225 \mathrm{~nm}$ (Fig. S11). The observation of similar PL bands indicated that the same emissive species contributed to the overall PL with different intensities. The PL intensity distribution was expected to depend considerably on the orientation and arrangement of the ligands relative to the Au NC surface, and thus, was also affected by the surface charge anisotropy, similar to the previously discussed absorption properties. Moreover, the PL spectra of AuMHA and AuMHA/HDT type A were independent of the excitation wavelength and showed matching excitation spectra (Figures 3a-c; Fig. S12), while the PL of AuMHA/HDT type B underwent a red shift accompanied by the appearance of a welldefined excitation band at $1040 \mathrm{~nm}$ (Figure 3d; Fig. S12). Observation of gel electrophoresis of type $A$ and type $B$ under SWIR cameras showed $\mathrm{PL}$ from $\mathrm{Au}_{23}$ and $\mathrm{Au}_{25}$ confirming that the origin of the SWIR PL was rather originating from the surface than from the core of the Au NCs. Therefore, we hypothesized that SWIR emission was probably originating from ligand-tometal charge-transfer bands (LMCT) and ligand-to-metal-metal charge transfer (LMMCT) via Au-S bonds. Types C and D displayed PL profiles similar to that of type B but with a weaker intensity at shorter SWIR wavelengths (Fig. S13). Apparently, the self-assembled network of type $D$ yielded no further influence on the PL properties, in contrast to its considerable effect on the absorption properties. Measurements of the PL decay kinetics revealed an increase in the intensity-averaged lifetime $\langle\tau\rangle_{\text {int. }}\left(\lambda_{\text {exc. }} 510 \mathrm{~nm} ; \lambda_{\text {em. }} 930 \pm 30 \mathrm{~nm}\right)-$ from $404 \mathrm{~ns}$ for AuMHA to $618 \mathrm{~ns}$ for AuMHA/HDT type B (Fig. S14)-when incorporating the co-ligand HDT. This increase in lifetime suggested multiple energy transfer processes involving $\mathrm{Au}$ ligand electronic states. The PL QY measurements of our Au NCS using IR125 as reference (see $\mathrm{SI}$ ) yielded PL QY values of $0.9 \%$, $6.1 \%$, and $4.7 \%$, for AuMHA, AuMHA/HDT type $A$, and AuMHA/HDT type $B$, respectively. This result demonstrated the tunability and enhancement of PL (here, by a factor of up to 6.8) via changing the surface charge of Au NCs.

We also measured the PL emission of the different Au NCs in solution using a SWIR camera (Princeton 640ST) and an excitation source at $\lambda_{\text {exc. }} 830 \mathrm{~nm}$ with different long pass filters (Fig. S15). Results confirmed the spectroscopic data with a higher contribution of SWIR PL at longer wavelengths above $1250 \mathrm{~nm}$ for the self-assembled Au NC structure.

\section{Conclusions}

In summary, by simply incorporating a short bidentate dithiol co-ligand on the Au NC surface, we could bathochromically shift and boost the absorption and photoluminescence of watersoluble $\mathrm{Au}$ NCs in the NIR-SWIR spectral window. This modification strongly affects the energy transfer processes involving surface electronic states and can lead to new discrete energy levels. The resulting Au NCs are among the brightest SWIR emitters reported so far with a largely red-shifted emission exceeding $1000 \mathrm{~nm}$, which is highly suitable for in vivo 
imaging. These findings will enable the rational design of the next generation of even brighter Au NCs and will pave the road to numerous new single and self-assembled metal nanoclusters for sensing or bio-medical applications and as optically active components in optoelectronic devices.

\section{Conflicts of interest}

There are no conflicts to declare.

\section{Acknowledgement}

XLG would like to thank Cancéropôle Lyon Auvergne RhôneAlpes (CLARA), Plan Cancer (C18038CS) and ARC (R17157CC) for their financial support. The TEM images were carried out as part of the DFG core facility project "Berlin Electron Microscopy Network (Berlin EM Network)". KDW acknowledges support from an Adolf-Martens fellowship granted by BAM, and URG acknowledges financial support by the German Research Council (DFG; MEranet project NanoHype; grant RE1203/20-1). The K-Alpha+ instrument was financially supported by the German Federal Ministry of Economics and Technology on the basis of a decision by the German Bundestag. The authors acknowledge support from the ICMG FR 2607 NMR platform and Béatrice Gennaro for NMR-DOSY measurements. KDW expresses his gratitude to Dr. J. Pauli for help with the fluorescence measurements. The TEM images were carried out as part of the DFG core facility project "Berlin Electron Microscopy Network (Berlin EM Network)".

\section{References}

1. G. Hong, A. Antaris and H. Dai, Nat. biom. Eng., 2017, 1, 0010 (0011-0022).

2. O. Bruns, T. Bischof, D. Harris, D. Franke, Y. Shi, L. Riedemann, A. Bartelt, F. Jaworski, J. Carr, C. Rowlands, M. Wilson, O. Chen, H. Wei, G. Hwang, D. Montana, I. Coropceanu, O. Achorn, J. Kloepper, J. Heeren, P. So, D. Fukumura, K. Jensen, R. Jain and M. Bawendi, Nat. biom. Eng., 2017, 1, 0056(0051-0011).

3. G. Hong, S. Diao, J. Chang, A. L. Antaris, C. Chen, B. Zhang, S. Zhao, D. N. Atochin, P. L. Huang, K. I. Andreasson, C. J. Kuo and H. Dai, Nat. Phot., 2014, 8, 723-730.

4. J. T. Robinson, G. Hong, Y. Liang, B. Zhang, O. K. Yaghi and H. Dai, J. Am. Chem. Soc., 2012, 134, 10664-10669.

5. M. Zhang, J. Yue, R. Cui, Z. Ma, H. Wan, F. Wang, S. Zhu, Y. Zhou, Y. Kuang, Y. Zhong, D. W. Pang and H. Dai, P.N.A.S., 2018, 115, 6590-6595.

6. A. L. Antaris, H. Chen, K. Cheng, Y. Sun, G. Hong, C. Qu, S. Diao Z. Deng, X. Hu, B. Zhang, X. Zhang, O. K. Yaghi, Z. R. Alamparambil, X. Hong, Z. Cheng and H. Dai, Nat. Mat., 2016, 15, 235-242.

7. G. Hong, S. Diao, A. L. Antaris and H. Dai, Chem. Rev., 2015, 115, 10816-10906.

8. D. Franke, D. K. Harris, O. Chen, O. T. Bruns, J. A. Carr, M. W. B. Wilson and M. G. Bawendi, Nat. Comm., 2016, 7.

9. G. Hong, J. T. Robinson, Y. Zhang, S. Diao, A. L. Antaris, Q. Wang and H. Dai, Angew. Chem. - Int. Ed., 2012, 51, 9818-9821.

10. Y. Chen, D. Montana, H. Wei, J. Cordero, M. Schneider, X. Le Guevel, O. Chen, O. Bruns and M. Bawendi, Nanolett., 2017, 17, 6330-6334.

11. X. Yuan, B. Zhang, Z. Luo, Q. Yao, D. T. Leong, N. Yan and J. Xie, Angew. Chem. - Int. Ed., 2014, 53, 4623-4627.

12. X. Yuan, N. Goswami, W. Chen, Q. Yao and J. Xie, Chem.Comm., 2016, 52, 5234-5237.
13. M. Waszkielewicz, J. Olesiak-Banska, C. Comby-Zerbino, F. Bertorelle, X. Dagany, A. K. Bansal, M. T. Sajjad, I. D. W. Samuel, Z. Sanader, M. Rozycka, M. Wojtas, K. Matczyszyn, V. BonacicKoutecky, R. Antoine, A. Ozyhar and M. Samoc, Nanoscale, 2018, 10, 11335-11341.

14. A. Sels, G. Salassa, F. Cousin, L. T. Lee and T. Bürgi, Nanoscale, 2018, 10, 12754-12762.

15. M. Zhou, C. Zeng, Y. Chen, S. Zhao, M. Y. Sfeir, M. Zhu and R. Jin, Nat.Comm., 2016, 7.

16. J. A. Jenkins, Y. Zhou, S. Thota, X. Tian, X. Zhao, S. Zou and J. Zhao, J.Phys. Chem. C, 2014, 118, 26276-26283.

17. D. E. Jiang, K. Nobusada, W. Luo and R. L. Whetten, ACS Nano, 2009, 3, 2351-2357.

18. M. De Nardi, S. Antonello, D. E. Jiang, F. Pan, K. Rissanen, M. Ruzzi, A. Venzo, A. Zoleo and F. Maran, ACS Nano, 2014, 8, 85058512.

19. C. M. Aikens, Acc. Chem. Res., 2018, 51, 3065-3073.

20. S. E. Crawford, C. M. Andolina, A. M. Smith, L. E. Marbella, K. A. Johnston, P. J. Straney, M. J. Hartmann and J. E. Millstone, J. Am. Chem. Soc., 2015, 137, 14423-14429.

21. S. Link, A. Beeby, S. FitzGerald, M. A. El-Sayed, T. G. Schaaff and R. L. Whetten, J. Phys. Chem. B, 2002, 106, 3410-3415.

22. G. Wang, T. Huang, R. W. Murray, L. Menard and R. G. Nuzzo, J. Am. Chem. Soc., 2005, 127, 812-813.

24. M. Zhou, C. Zeng, M. Y. Sfeir, M. Cotlet, K. lida, K. Nobusada and R. Jin, J. Phys. Chem. Lett., 2017, 8, 4023-4030. 